\title{
Prevalence of plasmid-mediated AmpC $\beta$-lactamases among uropathogenic Escherichia coli isolates in southwestern Iran
}

\author{
Nabi Jomehzadeh ${ }^{1}{ }^{\oplus}$, Khadijeh Ahmadi $^{1}{ }^{\oplus}$, Zahra Rahmani $^{2}$ (®) \\ ${ }^{1}$ Department of Microbiology, School of Medicine, Abadan University of Medical Sciences, Abadan, Iran \\ ${ }^{2}$ Abadan University of Medical Sciences, Abadan, Iran
}

Received: October 11, 2021

Revised: October 18, 2021

Accepted: November 15, 2021

Corresponding author:

Nabi Jomehzadeh

Department of Microbiology,

School of Medicine, Abadan

University of Medical Sciences,

Abadan 6313833177, Iran

E-mail:n.jomehzadeh@

abadanums.ac.ir

\section{ABSTRACT}

Objectives: This study was undertaken to evaluate AmpC $\beta$-lactamase-producing Escherichia coli urine isolates and to characterize the frequency of plasmid-mediated AmpC (pAmpC)encoding genes.

Methods: Antimicrobial susceptibility tests were performed using the disk diffusion technique. AmpC $\beta$-lactamase production was assessed with a phenotypic inhibitor-based method. The presence of $6 \mathrm{pAmpC}$-encoding cluster genes was detected by multiplex polymerase chain reaction (PCR).

Results: The proportion of antibiotic resistance of E. coli isolates ranged from $7.4 \%$ to $90.5 \%$, and more than half (51.6\%) of the total isolates were multidrug-resistant (MDR). Among the 95 E. coli isolates, 60 (63.2\%) were found to be cefoxitin-resistant, but only 14 (14.7\%) isolates were confirmed as AmpC $\beta$-lactamase-producers. In the PCR assay, pAmpC-encoding genes were found in 15 (15.8\%) isolates, and bla $a_{\mathrm{DHA}}$ was the most prevalent type. However, bla bla Acc genes were not detected in the isolates.

Conclusion: Our findings contributed valuable information concerning antibiotic resistance, confirmatory phenotypic testing for AmpC production, and $\mathrm{pAmpC} \beta$-lactamase gene content in E. coli isolates in southwestern Iran. The level of MDR recorded in AmpC-producing strains of this study was worrying; therefore, implementing strong infection control approaches to reduce the MDR burden is recommended.

Keywords: AmpC; Escherichia coli; Multidrug resistance; Urinary tract infections

\section{Introduction}

Escherichia coli is the most common cause of urinary tract infections worldwide. The increasing rates of antibiotic resistance among $E$. coli strains are worrying and have limited the empiric 
treatment options for these infections [1]. Beta-lactam antibiotics are of substantial importance in the treatment of infections due to their broad-spectrum antibacterial activity and selective toxicity, but their extensive usage over recent decades has led to resistance to them [2]. The production of AmpC $\beta$-lactamase enzymes, which confer resistance to a wide range of $\beta$-lactam antibiotics, excluding carbapenems and fourth-generation cephalosporins, is one of the key mechanisms of bacterial resistance to these types of antibiotics [3]. AmpC-type $\beta$-lactamases are in the $C$ group of Ambler and their common genotypes include bla $a_{\mathrm{FOX}}, b l a_{\mathrm{MOX}}, b l a_{\mathrm{CIT}}, b l a_{\mathrm{DHA}}, b l a_{\mathrm{EBC}}$, and bla $a_{\mathrm{ACC}}$. Originally, these genes were transferred chromosomally, but they can also be disseminated by plasmids or other mobile elements [4]. The acquisition of plasmid-mediated AmpC (pAmpC) $\beta$-lactamases genes by organisms such as $E$. coli has prompted major concerns because it leads to the emergence and wide dissemination of multidrug-resistant (MDR) strains that are clinically and epidemiologically very important [5]. The phenotypic diagnosis of AmpC-mediated resistance is difficult due to misleading results and may lead to treatment failure. In addition, the Clinical and Laboratory Standards Institute (CLSI) has not introduced any technique for the phenotypic detection of AmpC production in microorganisms [6]. Molecular tests such as polymerase chain reaction (PCR) to identify the presence of pAmpC genes, along with phenotypic methods, are essential. However, they are not routinely performed in clinical microbiology laboratories [7]. Awareness of the prevalence of AmpC $\beta$-lactamase-producing micro-organisms could be very valuable for achieving more accurate epidemiological results, as well as controlling their spread. Hence, this study was designed to assess the frequency of pAmpC $\beta$-lactamases in E. coli isolates causing urinary tract infections, utilizing both phenotypic and genotypic methods.

\section{Materials and Methods}

\section{Study Setting and Bacterial Isolation}

In a 7-month period (from August 2020 to March 2021), 95 consecutive non-repetitive urine isolates of $E$. coli were obtained from clinical diagnostic laboratories and medical centers affiliated with Abadan University of Medical Sciences in southwestern Iran. Isolates were accurately identified through routine microbiological diagnostic tests [8]. The confirmed isolates were stored in a trypticase soy broth (Merck Co., Darmstadt, Germany) containing 20\% glycerol at $-70^{\circ} \mathrm{C}$ until antibiograms and molecular tests.

\section{Antibiotic Susceptibility Testing}

Antibiograms of the confirmed E. coli isolates for 10 standard antibiotics (Roscoe, Taastrup, Denmark), including tetracycline

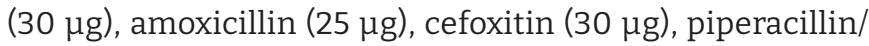
tazobactam $(100 / 10 \mu \mathrm{g})$, cefpodoxime $(30 \mu \mathrm{g})$, cephalothin $(30 \mu \mathrm{g})$, ceftriaxone $(30 \mu \mathrm{g})$, amikacin $(30 \mu \mathrm{g})$, ceftizoxime $(30 \mu \mathrm{g})$, colistin $(10 \mu \mathrm{g})$, were carried out using the standard disk diffusion method as recommended by the CLSI [9]. The $E$. coli ATCC 25922 strain was used for quality control. In this study, 8 different classes of antibiotics including penicillins; first-, second-, and third-generation cephalosporins; tetracyclines; aminoglycosides; polymyxins; and $\beta$-lactamase inhibitors were used. Isolates that were resistant to at least 1 agent in 3 or more classes of antimicrobials were considered MDR.

\section{Screening and Confirmatory Testing of AmpC Production}

All isolates were first screened for the probable production of AmpC $\beta$-lactamases by placing a cefoxitin disk $(30 \mu \mathrm{g})$ on Mueller-Hinton agar (Merck Co.) [10]. Isolates that conferred an inhibition zone diameter of $<18 \mathrm{~mm}$ were considered potential producers of AmpC and subjected to the confirmatory phenotypic test. Screening-positive AmpC producers were confirmed by an inhibitor-based method on a disk containing boronic acid [11]. Briefly, a lawn culture of the tested isolates was made on a Mueller-Hinton agar plate using 0.5 McFarland solutions. Two disks of cefoxitin $(30 \mu \mathrm{g})$ with and without phenylboronic acid $(400 \mu \mathrm{g})$ was placed onto the agar surface and the results were interpreted. If the growth inhibition zone around the antibiotic with phenylboronic acid compared to the disk containing only cefoxitin was $5 \mathrm{~mm}$ or greater, the isolate was considered to be an $\mathrm{AmpC}$ producer.

\section{Detection of pAmpC Genes}

The genomic DNA of the isolates was extracted using a commercial extraction kit (SinaClon BioScience Co., Tehran, Iran) following the manufacturer's instructions. The detection of 6 different families of pAmpC $\beta$-lactamases, including bla $a_{\mathrm{FOX}}$, bla $_{\mathrm{MOX}}$, bla $_{\mathrm{CIT}}, b l a_{\mathrm{DHA}}$, bla $_{\mathrm{EBC}}$, and bla $a_{\mathrm{ACC}}$, was performed as previously described by Perez-Perez and Hanson [12]. All primers were synthesized and provided by SinaClon (SinaClon BioScience Co.).

\section{Ethics Approval}

This research was approved by the local ethics committee of the Abadan University of Medical Sciences (No. IR.ABADANUMS. REC.1399.070), Abadan, Iran, and was conducted in accordance with the Declaration of Helsinki. Written informed consent was obtained from all the participants. 


\section{Results}

In this study, the highest antibiotic resistance was observed first towards amoxicillin (90.5\%) and then to cephalothin (81.1\%). Of the $95 \mathrm{E}$. coli isolates tested, 60 (63.2\%) were resistant to cefoxitin, while 9 (9.5\%) showed intermediate resistance. Alternatively, of 69 (72.6\%) isolates insensitive to cefoxitin, 58 (61.1\%), 55 (57.9\%), and 52 (54.7\%) isolates were resistant to ceftriaxone, cefpodoxime, and ceftizoxime, respectively. The isolates showed the highest susceptibility to colistin (92.6\%), followed by piperacillin/tazobactam (82.1\%) and amikacin (62.1\%) (Table 1). Among the all studied isolates, 49 (51.6\%) exhibited MDR phenotypes, with 8 different profiles. The group VII resistance pattern of strains (ceftizoxime, cefoxitin, ceftriaxone, cefpodoxime, amoxicillin, cephalothin) was the most prevalent (20.4\%) (Table 2). Of all 95 E. coli isolates investigated for $\beta$-lactamases, 60 (63.2\%) were suspected to be AmpC producers by the disk agar diffusion cefoxitin screening test. After performing the confirmatory phenotypic test, the frequency of confirmed isolates producing AmpC $\beta$-lactamase was determined to be $14.7 \%$ (14/95). Concerning the antibiogram results, the resistance rate of AmpC $\beta$-lactamase-producing strains to all antimicrobial agents was higher than that of $81 \mathrm{AmpC}-$ negative strains (Figure 1). Multiplex PCR for pAmpC genes revealed that 8 (8.4\%), 4 (4.2\%), and $3(3.2 \%)$ of E. coli strains tested positive for the $b l a_{\mathrm{DHA}}, b l a_{\mathrm{CIT}}$, and $b l a_{\mathrm{EBC}}$ genes, respectively. The other 3 genotypes $\left(b l a_{\text {MOX }}\right.$, bla $a_{\text {Fox }}$, and $\left.b l a_{\text {AcC }}\right)$ were not detected in any strains (Table 3 ).

\section{Discussion}

The growing frequency of antibiotic resistance as a primary public health concern in developing and undeveloped countries has resulted in the failure to treat various infections, higher handling costs, limited therapeutic choices, and increased mortality and morbidity [13]. pAmpC $\beta$-lactamases have become increasingly significant from a therapeutic standpoint, and their identification will be useful for both monitoring and epidemiological and infection control strategies [5]. In this study, the level of antibiotic resistance of $E$. coli isolates ranged from $7.4 \%$ to $90.5 \%$. Antimicrobial susceptibility testing revealed that the bacterial isolates studied had relatively high resistance to $\beta$-lactam antibiotics (amoxicillin, cephalothin, cefoxitin, ceftriaxone, cefpodoxime, and ceftizoxime). Meanwhile,

Table 1. The antimicrobial susceptibility profile of all isolates $(n=95)$

\begin{tabular}{lccc}
\hline Antimicrobials & Resistant (\%) & Intermediate (\%) & Susceptible (\%) \\
\hline Ceftriaxone & $58(61.1)$ & $7(7.4)$ & $30(31.6)$ \\
Cefoxitin & $60(63.2)$ & $9(9.5)$ & $26(27.4)$ \\
Cefpodoxime & $55(57.9)$ & $2(2.1)$ & $38(40.0)$ \\
Cephalothin & $77(81.1)$ & $4(4.2)$ & $14(14.7)$ \\
Ceftizoxime & $52(54.7)$ & $4(4.2)$ & $39(41.1)$ \\
Amikacin & $27(28.4)$ & $9(9.5)$ & $59(62.1)$ \\
Tetracycline & $52(54.7)$ & $3(3.2)$ & $40(42.1)$ \\
Amoxicillin & $86(90.5)$ & 0 & $9(9.5)$ \\
Piperacillin/tazobactam & $16(16.8)$ & $1(1.1)$ & $78(82.1)$ \\
Colistin & $7(7.4)$ & 0 & $88(92.6)$ \\
\hline
\end{tabular}

Table 2. Multidrug-resistance patterns of isolates $(n=49)$

\begin{tabular}{lll}
\hline Resistance pattern & \multicolumn{1}{c}{ Resistance phenotypes } & $n(\%)$ \\
\hline I & KF, AN, AMX & $9(18.4)$ \\
II & CZX, CRO, FOX, TZP & $3(6.1)$ \\
III & CZX, FOX, CRO, CPD, TET, AMX & $8(16.3)$ \\
IV & CRO, KF, AN, AMX, FOX & $5(10.2)$ \\
V & CZX, TZP, FOX, AN, TET, AMX, CPD & $7(14.3)$ \\
VI & CZX, CRO, CPD, CS, AMX, TZP, AN, TET & $1(2.0)$ \\
VII & CZX, FOX, CRO, CPD, AMX, KF & $10(20.4)$ \\
VIII & CZX, FOX, CRO, CPD, TET, AMX, KF, AN, TZP & $6(12.2)$ \\
\hline
\end{tabular}

KF, cephalothin; AN, amikacin; AMX, amoxicillin; CZX, ceftizoxime; CRO, ceftriaxone; FOX, cefoxitin; TZP, piperacillin/tazobactam; CPD, cefpodoxime; TET, tetracycline; CS, colistin. 


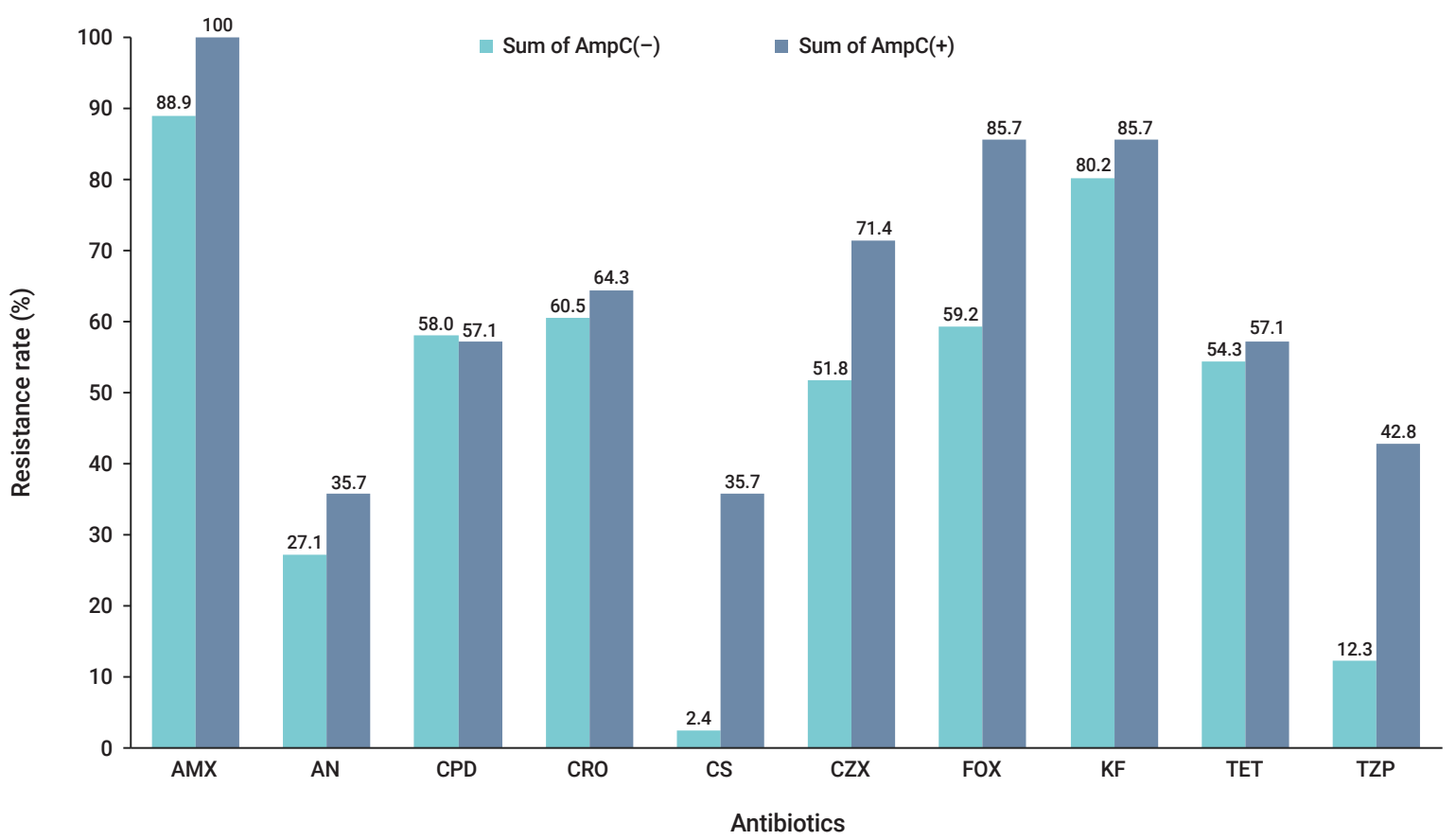

Figure 1. Comparison of antibiotic resistance among AmpC-positive and -negative strains (\%). AMX, amoxicillin; AN, amikacin; CPD, cefpodoxime; CRO, ceftriaxone; CS, colistin; CZX, ceftizoxime; FOX, cefoxitin; KF: cephalothin; TET, tetracycline; TZP, piperacillin/tazobactam.

Table 3. Distribution of plasmid-mediated AmpC $\beta$-lactamase genes among Escherichia coli strains $(n=95)$

\begin{tabular}{ll}
\hline AmpC positive & $n(\%)$ \\
\hline Cefoxitin screening test & $60(63.2)$ \\
Confirmatory phenotypic test & $14(14.7)$ \\
Multiplex polymerase chain reaction & $15(15.8)$ \\
AmpC gene & \\
$b / a_{\mathrm{DHA}}$ & $8(8.4)$ \\
$b / a_{\mathrm{CIT}}$ & $4(4.2)$ \\
$b / a_{\mathrm{EBC}}$ & $3(3.2)$ \\
$b / a_{\mathrm{MOX}}$ & 0 \\
bla $_{\mathrm{FOX}}$ & 0 \\
$b / a_{\mathrm{ACC}}$ & 0 \\
& \\
\hline
\end{tabular}

they had the highest susceptibility to colistin, piperacillin/ tazobactam, and amikacin. Comparable results were reported by some previous studies from Nepal [13], India [14], and Iran [15]. As shown in Figure 1, AmpC producers exhibited significantly higher resistance rates than AmpC-negative strains. This observation extensively corroborated the findings of previous authors, associating AmpC production with increasing resistance to diverse antimicrobial classes [16-18]. Antimicrobial resistance is linked to the increasing spread of MDR strains, and since infections caused by these organisms are extremely difficult to treat, recognition and awareness of their prevalence in the community are crucial
[19]. In the present study, almost half of the E. coli isolates (49, 51.6\%) were found to be MDR, which is in line with the results of other studies carried in Iran [20] and Gabon [1] and contrary to that of Bala et al. [14]. There are several reasons for the reduction in sensitivity towards newer generations of antibiotics, including bacterial production of $\beta$-lactam ring hydrolyzing enzymes such as extendedspectrum $\beta$-lactamases, AmpC $\beta$-lactamases, and metallo$\beta$-lactamases [21]. Although various screening and confirmatory phenotypic methods for AmpC $\beta$-lactamase have been introduced, most of them are not suitable for routine use in diagnostic laboratories. However, the inhibitor-based method, which serves as a reliable confirmatory marker with acceptable negative predictive values, has been employed by many researchers to detect AmpC production [17]. As expected, in the present study, screening and confirmatory phenotypic tests yielded different results, and the production of AmpC $\beta$-lactamase was confirmed in only $14.7 \%$ (14/95) of cefoxitin-resistant isolates. Studies conducted in Spain (14.2\%) [22], Nigeria (15.2\%) [23], and Iran (15.1\%) [5] have reported equivalent frequencies. However, the frequency rate of AmpC production in the present study was lower than those found in other studies conducted in India [14], Bahrain [24], Iran [25], and Egypt [6], which reported rates of $47.1 \%, 87 \%$, $54.4 \%$, and $73.4 \%$, respectively. There are several reasons 
for this discrepancy in the findings, including differences in the diagnostic methods employed, geographical location, study participants, and the study period. Various studies have been conducted on the prevalence of the pAmpC genes at different time points and in different countries, which can provide valuable information about AmpCtype resistance over time and its course. According to the multiplex PCR assay results of this study, 3 types of PAmpC cluster genes ( $b l a_{\mathrm{CIT}}, b l a_{\mathrm{DHA}}$, and $\left.b l a_{\mathrm{DHA}}\right)$ were detected in 15 isolates (15.8\%). The prevalence of the bla $a_{\mathrm{DHA}}, b l a_{\mathrm{CIT}}$, and $b l a_{\mathrm{EBC}}$ genes in E. coli isolates was also found to be $8.4 \%, 4.2 \%$, and $3.2 \%$, respectively. These results were in accordance with a previous study reported by Kazemian et al. [26], from Iran. Although some studies have reported the coexistence of bla $a_{\mathrm{DHA}}, b l a_{\mathrm{EBC}}$, and bla $a_{\mathrm{CIT}}$ in E. coli isolates [15,25,27], only 1 pAmpC gene family was detected in the strains of our study. Some limitations of this study included the lack of data on the molecular typing of the strains and the sequencing of pAmpC cluster genes. In addition, due to a lack of funding, only the presence of pAmpC genes was targeted, and other potential associates of cefoxitin resistance, such as chromosomal hyperproduction or purine loss mutations, were not investigated.

\section{Conclusion}

This is the first study to investigate pAmpC $\beta$-lactamases using phenotypic and molecular methods among patients in southwestern Iran, and our research results could provide useful information to support the development of antimicrobial strategies for better infection control in healthcare facilities. In this study, a significant percentage of MDR was observed among AmpC-producing strains. The PCR results also showed that $15.8 \%$ of phenotypically confirmed isolates harbored pAmpC $\beta$-lactamase genes $\left(b l a_{\mathrm{DHA}}, b l a_{\mathrm{CIT}}\right.$, and $b l a_{\mathrm{EBC}}$ ), and the most prevalent genotype belonged to bla $_{\mathrm{DHA}}(8.4 \%)$.

\section{Notes}

\section{Ethics Approval}

This research was approved by the local ethics committee of the Abadan University of Medical Sciences (No. IR.ABADANUMS.REC.1399.070), Abadan, Iran, and was conducted in accordance with the Declaration of Helsinki. Written informed consent was obtained from all the participants.

\section{Conflicts of Interest}

The authors have no conflicts of interest to declare.

\section{Funding}

This study was financially supported by the Vice-Chancellor for Research of Abadan University of Medical Sciences, Abadan, Iran (Grant No. 99T.829).

\section{Availability of Data}

All data generated or analyzed during this study are included in this published article. For other data, these may be available through the corresponding author upon reasonable request.

\section{Authors' Contributions}

Conceptualization: NJ; Data curation: NJ; Formal analysis: NJ; Investigation: all authors; Methodology: KA, ZR; Project administration: NJ; Resources: all authors; Software: all authors; Supervision: NJ; Validation: all authors; Visualization: all author; Writing-original draft: NJ; Writing-review \& editing: NJ. All the authors reviewed and approved the final draft, and are responsible for all aspects of the work.

\section{Additional Contributions}

This research was derived from the general physician thesis by Zahra Rahmani under the supervision of Dr. N Jomehzadeh, which was approved by Abadan University of Medical Sciences, Abadan, Iran. The authors appreciate all those who contributed directly or indirectly to this research.

\section{References}

1. Mouanga Ndzime Y, Onanga R, Kassa Kassa RF, et al. Epidemiology of community origin Escherichia coli and Klebsiella pneumoniae uropathogenic strains resistant to antibiotics in Franceville, Gabon. Infect Drug Resist 2021;14:585-94.

2. Dalhoff A. Selective toxicity of antibacterial agents-still a valid concept or do we miss chances and ignore risks? Infection 2021; 49:29-56

3. Najjuka CF, Kateete DP, Lodiongo DK, et al. Prevalence of plasmidmediated AmpC beta-lactamases in Enterobacteria isolated from urban and rural folks in Uganda. AAS Open Res 2020;3:62.

4. Santiago GS, Goncalves D, da Silva Coelho I, et al. Conjugative plasmidic AmpC detected in Escherichia coli, Proteus mirabilis and Klebsiella pneumoniae human clinical isolates from Portugal. Braz J Microbiol 2020;51:1807-12.

5. Saffar H, Asgari Niaraki N, Ghahroudi Tali A, et al. Prevalence of AmpC $\beta$-lactamase in clinical isolates of Escherichia coli, Klebsiella spp., and Proteus mirabilis in a Tertiary Hospital in Tehran, Iran. Jundishapur J Microbiol 2016;9:e39121.

6. Helmy MM, Wasfi R. Phenotypic and molecular characterization of plasmid mediated AmpC $\beta$-lactamases among Escherichia coli, Klebsiella spp., and Proteus mirabilis isolated from urinary tract infections in Egyptian hospitals. Biomed Res Int 2014;2014:171548.

7. Chakraborty A, Adhikari P, Shenoy S, et al. Characterization of plasmid mediated AmpC producing Escherichia coli clinical isolates from a tertiary care hospital in South India. Indian J Pathol Microbiol 2014;57:255-8.

8. Jomezadeh N, Farajzadeh A, Javaherizadeh $\mathrm{H}$, et al. The prevalence of asymptomatic bacteriuria in infants and children. Pak Pediatr J 2011;35:13-6.

9. Clinical Laboratory Standards Institute (CLSI). Performance standards for antimicrobial susceptibility testing, 30th ed. CLSI supplement 100. Wayne: CLSI; 2020. 
10. Polsfuss S, Bloemberg GV, Giger J, et al. Practical approach for reliable detection of AmpC beta-lactamase-producing Enterobacteriaceae. J Clin Microbiol 2011;49:2798-803.

11. Coudron PE. Inhibitor-based methods for detection of plasmidmediated AmpC beta-lactamases in Klebsiella spp., Escherichia coli, and Proteus mirabilis. J Clin Microbiol 2005;43:4163-7.

12. Perez-Perez FJ, Hanson ND. Detection of plasmid-mediated AmpC beta-lactamase genes in clinical isolates by using multiplex PCR. J Clin Microbiol 2002;40:2153-62.

13. Aryal SC, Upreti MK, Sah AK, et al. Plasmid-mediated AmpC $\beta$-lactamase CITM and DHAM genes among gram-negative clinical isolates. Infect Drug Resist 2020;13:4249-61.

14. Bala R, Singh VA, Gupta N, et al. Prevalence, multidrug-resistance and risk factors for AmpC $\beta$-lactamases producing Escherichia coli from hospitalized patients. J Infect Dev Ctries 2020;14:1466-9.

15. Robatjazi S, Nikkhahi F, Niazadeh M, et al. Phenotypic identification and genotypic characterization of plasmid-mediated AmpC $\beta$-lactamase-producing Escherichia coli and Klebsiella pneumoniae isolates in Iran. Curr Microbiol 2021;78:2317-23.

16. Ibrahim ME, Abbas M, Al-Shahrai AM, etal. Phenotypic characterization and antibiotic resistance patterns of extended-spectrum $\beta$-lactamaseand AmpC $\beta$-lactamase-producing gram-negative bacteria in a referral hospital, Saudi Arabia. Can J Infect Dis Med Microbiol 2019; 2019:6054694.

17. Akya A, Elahi A, Chegene Lorestani R, et al. Antibiotic resistance and phenotypically and genotypically AmpC beta-lactamases among Escherichia coli isolates from outpatients. J Gorgan Univ Med Sci 2019;20:108-14.

18. Tekele SG, Teklu DS, Tullu KD, et al. Extended-spectrum betalactamase and AmpC beta-lactamases producing gram negative bacilli isolated from clinical specimens at International Clinical Laboratories, Addis Ababa, Ethiopia. PLoS One 2020;15:e0241984.

19. Pokharel S, Raut S, Adhikari B. Tackling antimicrobial resistance in low-income and middle-income countries. BMJ Glob Health 2019; 4:e002104.
20. Koshesh M, Mansouri S, Hashemizadeh Z, et al. Identification of extended-spectrum $\beta$-lactamase genes and AmpC- $\beta$-lactamase in clinical isolates of Escherichia coli recovered from patients with urinary tract infections in Kerman, Iran. Arch Pediatr Infect Dis 2017;5:e37968.

21. Kakoullis L, Papachristodoulou E, Chra P, et al. Mechanisms of antibiotic resistance in important gram-positive and gram-negative pathogens and novel antibiotic solutions. Antibiotics (Basel) 2021; 10:415.

22. Gomara M, Lopez-Calleja AI, Iglesia B, et al. Detection of carbapenemases and other mechanisms of enzymatic resistance to $\beta$-lactams in Enterobacteriaceae with diminished susceptibility to carbapenems in a tertiary care hospital. Enferm Infecc Microbiol Clin (Engl Ed) 2018;36:296-301.

23. Ogefere HO, Osikobia JG, Omoregie R. Prevalence of AmpC $\beta$-lactamase among Gram-negative bacteria recovered from clinical specimens in Benin City, Nigeria. Trop J Pharm Res 2016;15:1947-53.

24. Joji RM, Al-Mahameed AE, Jishi TA, et al. Molecular detection of plasmid-derived AmpC $\beta$-lactamase among clinical strains of Enterobacteriaceae in Bahrain. Ann Thorac Med 2021;16:287-93.

25. Motamedifar M, Mohebi S, Hadadi M, et al. The prevalence of ESBL and AmpC $\beta$-lactamases in uropathogenic isolates of Escherichia coli in a tertiary care hospital in Southwest Iran. Gene Rep 2020;20: 100747.

26. Kazemian H, Heidari H, Ghanavati R, et al. Phenotypic and genotypic characterization of ESBL-, AmpC-, and carbapenemase-producing Klebsiella pneumoniae and Escherichia coli Isolates. Med Princ Pract 2019;28:547-51.

27. Mohamudha PR, Harish BN, Parija SC. Molecular description of plasmid-mediated AmpC $\beta$-lactamases among nosocomial isolates of Escherichia coli \& Klebsiella pneumoniae from six different hospitals in India. Indian J Med Res 2012;135:114-9. 